\title{
A Management Model of Small- and Medium-Sized Enterprises Based on Deep Learning Algorithm
}

\author{
Junshu Wang \\ Xinxiang Radio and Television University, Xinxiang City, Henan Province 453000, China \\ Correspondence should be addressed to Junshu Wang; 2002020129@st.btbu.edu.cn
}

Received 27 August 2021; Revised 19 October 2021; Accepted 25 October 2021; Published 24 November 2021

Academic Editor: Rahman Ali

Copyright (C) 2021 Junshu Wang. This is an open access article distributed under the Creative Commons Attribution License, which permits unrestricted use, distribution, and reproduction in any medium, provided the original work is properly cited.

\begin{abstract}
The traditional management method of small- and medium-sized enterprises (SMEs) has the problem of poor turnover prediction effect. Therefore, this paper proposes a management model of SMEs based on the deep learning algorithm. Firstly, the proposed system analyzes the characteristics of SMEs, and based on the deep learning algorithm, the gating structure of the management of SMEs is designed. The internal structure diagram of the long- and short-term neural network is given, and the circulating neural network model of the management of SMEs is constructed. Finally, the experimental indexes of the management model of SMEs are designed, and the comparative experiments are carried out. The experimental results show that the proposed method is more accurate in predicting the turnover of SMEs and has better management effect on them.
\end{abstract}

\section{Introduction}

Small- and medium-sized enterprises play an important role in social and economic development; different from foreign countries, China's SMEs grew up in a specific historical environment; most of the enterprises have still extensive management, lack of competitiveness in the market; especially after China's accession to the WTO (World Trade Organization), the management problem is increasingly prominent. Undoubtedly, enterprise informatization construction provides a new way for SMEs to develop, through which advanced management ideas and information technology can be introduced [1]. In recent years, with the gradual improvement of the status of SMEs in the national economy, more and more scholars and enterprises begin to pay attention to the theoretical research and application construction of SME informatization [2].

Small- and medium-sized enterprise is a relatively vague concept; generally speaking, the unified and adaptive standard to all industries definition does not exist. An overview of the definition standards of SMEs in various countries (or regions) in the world shows that not only the concept of SMEs is not unified but even the definition standards of SMEs in a country are not invariable [3]. Each country (or region) according to their own stage of economic development, level, and status of SMEs is defined accordingly; the same country (or region) will adjust its definition of SMEs accordingly with the continuous development of its economy. Generally speaking, the definition of SMEs can be measured from both qualitative and quantitative perspectives [4]. In order to improve the management effect of SMEs, this paper proposes a smalland medium-sized enterprise management model based on the deep learning algorithm.

\section{Analysis of Characteristics of SMEs}

In recent years, the development of small- and mediumsized enterprises in China is very rapid and has become the main force to promote the development of modern market economy. SMEs as a whole, compared with large enterprises, have the following characteristics:

(1) Large Quantity and Wide Range, Simple Mechanism, and High Efficiency. The industry distribution range of SMEs is very wide, and each industry has SMEs distributed among them. In addition to special industries controlled by the state and some industries with high technology and capital content, 
SMEs almost involve all industries and fields. And SMEs due to a wide variety of products, business projects, and wide range of services can fill the needs of all levels of society. However, most SMEs adopt the centralized system, without too many management levels, and the internal organization and operation structure are relatively simple. The business operator is not only the strategic formulation and decision maker but also the direct implementer, often holding several positions, thus giving full play to the advantages of high work efficiency, fast information transmission, and easy distortion [5].

(2) The Strain Ability Is Strong, the Management Mechanism Is Flexible. The "threshold" of capital and technology required by SMEs to start a business is low, production is easy to start, the results are quick, and it is easier to enter the market. Enterprise management means are flexible, can timely change the direction of production and management, adjust the product structure, and even change industries according to market changes; this feature enables SMEs to provide the market with urgently needed products. However, large enterprises are less flexible than SMEs, so it is not easy for them to change their production [6].

(3) Insufficient Funds, Small Scale, Division of Labor, and Cooperation Level Is Not High. SMEs have a small number of employees and small production scale, lack of sufficient capital accumulation; venture capital and working capital are relatively scarce compared with large enterprises, and financing channels are limited, mainly through relatives and friends borrowing and internal accumulation, it is not easy to find loan guarantee, and the financing is very difficult. This is because banks or social financing institutions generally believe that SMEs are weak in strength, low in credit, and relatively risky in lending, and there are also some defects in their financial management [7].

(4) The Staff Quality Is Low, the Management System Is Lack, and the Market Competitiveness Is Not Strong. The overall quality of SMEs personnel is low, lack of technical and management personnel. Needless to say, the heads of enterprises and even the personnel in the departments specializing in management have received no full-time training. The concept of management personnel is outdated, and there is the phenomenon of family management. The randomness of management is strong lacks institutionalized, standardized, and procedural management model. Personnel choose nepotism; the punishment system is not transparent and unable to mobilize the enthusiasm and creativity of employees. Without paying attention to talent development, it is difficult for individuals to have opportunities for promotion and development, and talent shortage and brain drain coexist [8]. Smalland medium-sized enterprises have disadvantages in terms of management level, capital accumulation, production scale, and personnel quality. As a result, its costs have increased, labor productivity has decreased, and product research and development capabilities have become weaker.

2.1. The Significance of Enterprise Informatization Solution. Enterprise information solutions are planning, organizing, controlling, and managing enterprise informatization implementation of the systematic method. It comprehensively considers various factors in the planning and implementation of the information system (including the current enterprise production management requirements to be met, the bottleneck which restricts the development of the enterprise, the enterprise development direction in the future, the enterprise existing information technology base, and the enterprise personnel quality) to establish a comprehensive enterprise information planning, establish a gradual development and evolution of information system framework, and give the implementation of the right way, so as to ensure enterprise information work is smooth, efficient, and low cost and ensure that the established information technology system can properly adjust and connect to the business structure and business strategy, for the enterprise production and management to provide effective support. Enterprise informatization schemes are divided into application solutions, technical solutions, and overall solutions [9]. The enterprise informatization scheme has the characteristics of generality, particularity, foresight, hierarchy, and inheritance. Generality means that the basic idea, basic method, and basic principle of enterprise informatization scheme are not defined for a specific enterprise but are applicable to all enterprises. At the same time, due to the specific situation of each enterprise, specific informatization solutions must have their own characteristics, so enterprise informatization solutions are also special [10]. Forward-looking means that enterprise informatization plan is the work plan and implementation way of enterprise informatization formulated by advanced methods and technologies under the guidance of advanced theories. It should not only solve the problems existing in the current information work of enterprises but also plan its implementation steps from the perspective of long-term development. Hierarchy means that the overall solution of enterprise informatization has different levels. The focus of the overall solution varies with the hierarchy. Inheritance means that low-level solutions support high-level enterprise informatization solutions [11].

\section{Small- and Medium-Sized Enterprise Data Division Based on Deep Learning}

3.1. Design of Gate Control Structure for SME Management. In modern advanced Chinese dictionaries, the word pattern refers to a standard pattern of things, such as a 
development pattern. The establishment of pattern theory is usually based on the occurrence of repetitive problems. The general practice is to first find the recurring problems in a specific environment, then find the solution to the problem, and finally describe the typical problems and summarize the corresponding solution, in order to form a pattern. In this way, existing solutions can be used countless times when similar problems are encountered, without redundant duplication [12].

LSTM is another common recurrent neural network, which is a more powerful and general version than GRU. In LSTM, we still have a small- and medium-sized enterprise data update $\Gamma_{u}$, and $\Gamma_{u}=\sigma\left(W_{u}\left[a^{\langle t-1\rangle}, x^{\langle t\rangle}\right]+b_{u}\right)$, SME data control is not only composed of SME data, but also a new Chinese SME data called Forgotten. Set $\Gamma_{f}$ as the parameter value, and $\Gamma_{f}=\sigma\left(W_{f}\left[a^{\langle t-1\rangle}, x^{\langle t\rangle}\right]+b_{f}\right)$. Then added a new output function, namely, $\Gamma_{o}=\sigma\left(W_{o}\left[a^{\langle t-1\rangle}, x^{\langle t\rangle}\right]+b_{o}\right)$. The updated value of memory cells in the SME data is changed to $c^{\langle t\rangle}=\Gamma_{u} * c^{\prime\langle t\rangle}+\Gamma_{f} * c^{\langle t-1\rangle}$. The use of separate update and forgetting gates gives the memory cell the option to maintain the old $c^{\langle t-1\rangle}$ or add the new value $c^{\prime\langle t\rangle}$. The complete formula of LSTM for SMEs is shown in the following equation:

$$
\begin{aligned}
c^{\prime\langle t\rangle} & =\tanh \left(W_{c}\left[a^{\langle t-1\rangle}, x^{\langle t\rangle}\right]+b_{c}\right), \\
\Gamma_{u} & =\sigma\left(W_{c}\left[a^{\langle t-1\rangle}, x^{\langle t\rangle}\right]+b_{u}\right), \\
\Gamma_{f} & =\sigma\left(W_{f}\left[a^{\langle t-1\rangle}, x^{\langle t\rangle}\right]+b_{f}\right), \\
\Gamma_{o} & =\sigma\left(W_{o}\left[a^{\langle t-1\rangle}, x^{\langle t\rangle}\right]+b_{o}\right), \\
c^{\langle t\rangle} & =\Gamma_{u} * c^{\langle\langle t\rangle}+\Gamma_{f} * c^{\langle t-1\rangle}, \\
a^{\langle t\rangle} & =\Gamma_{o} * c^{\langle t\rangle} .
\end{aligned}
$$

The internal structure of LSTM is shown in Figure 1.

In the history of deep learning, LSTM appeared earlier, and GRU was invented after LSTM. GRU originates from simplification on a more complex LSTM [14]. The advantage of GRU lies in its simple structure, which makes it easier to build a larger network, while LSTM is more powerful and flexible. It is also the gating structure that is first considered for deep learning tasks in the management of SMEs.

3.2. A Recurrent Neural Network Model for SMEs Management. The goal of SME turnover forecast is to forecast future sales volume through various characteristic values (influencing factors) and known SME operating values. The formula for SME turnover prediction is similar to the formula for autoregression prediction. The input is defined as all characteristic sets $\left\{x_{t}\right\}_{t=1}^{T}=\left\{x_{1}, x_{2}, \ldots, x_{T}\right\}$, and the corresponding SME turnover set is $\left\{y_{t}\right\}_{t=1}^{T}=\left\{y_{1}, y_{2}, \ldots, y_{T}\right\}$ [15]. At time $t, x_{t} \in R^{n}$, where $n$ is the characteristic dimension, $T$ is the entire time stage, and the output of business forecast of SMEs is the next turnover value of $\Delta$ after $T$ time stage, which can be expressed as the following formula:

$$
\left\{y^{\prime}\right\}_{t=T+1}^{T+\Delta}=\left\{y_{T+1}^{\prime}, y_{T+2}^{\prime}, \ldots,,_{T+\Delta}^{\prime}\right\},
$$

where $\Delta$ is determined according to the target of business forecast of SMEs, it is assumed that $\Delta \ll T$, and $\left\{x_{t}\right\}_{t=T+1}^{T+\Delta}$ is an unknown factor in the forecast stage [16].

Compared with the traditional autoregressive model, the performance of the SME business prediction model is different because the scalar value and the future are not characteristic. Therefore, we model the turnover forecast of SMEs with characteristics as follows:

$$
\left\{y_{t}\right\}_{t=T+1}^{T+\Delta}=F\left(\left\{x_{t}\right\}_{t=1}^{T},\left\{y_{t}\right\}_{t=1}^{T}\right),
$$

where $\left\{x_{t}\right\}_{t=1}^{T}$ is the characteristics from time 1 to $T,\left\{y_{t}\right\}_{t=1}^{T}$ is the historical business information of SMEs, $\left\{y_{t}\right\}_{t=T+1}^{T+\Delta}$ is the value to be predicted, and $F(\cdot)$ is the nonlinear mapping method to be learned.

The activation function $\sigma$ is the logistic Sigmoid function.

\section{Management Model of SMEs Based on Deep Learning}

For the Triage Amalgamated Dermoscopic Algorithm (TADA) model [17], the definition of the business forecast task for small and medium enterprises shows that the predicted scalar value has no characteristics in the future, as shown in formula (4). Let us say that $\left\{x_{t}\right\}_{t=T+1}^{T+\Delta}$ is an unknown factor at the prediction stage. For the sales data of agricultural products enterprises, the future temperature, sunshine and precipitation, and other factors can be learned from the weather forecast. Although there is a certain error in the weather forecast, with the advancement of science and technology, the accuracy of the weather forecast is getting higher and higher, assuming that the accuracy of the weather forecast is 0.8 . Therefore, when predicting $\left\{y_{t}\right\}_{t=T+1}^{T+\Delta}$, there can be other features besides $\left\{x_{t}\right\}_{t=1}^{T},\left\{y_{t}\right\}_{t=1}^{T}$, which may have probabilities of $1,0.98$, or 0.8 , but these features are accurate with high probability. Simply discarding this part of the information may reduce the accuracy of the prediction.

Incorporating this information into the SME turnover forecasting task can be very helpful. Based on the above ideas to improve the original TADA model, for SMEs business forecasting problem to define, improving the small- and medium-sized enterprise business model for prediction of the input is defined as all the characteristics of the set $\left\{x_{t}\right\}_{t=1}^{T}=\left\{x_{1}, x_{2}, \ldots, x_{T}\right\}$ and at the same time of the smalland medium-sized enterprise turnover set $\left\{y_{t}\right\}_{t=1}^{T}=\left\{y_{1}, y_{2}, \ldots, y_{T}\right\}$, and $T+1$, as $T+\Delta$ delta between small- and medium-sized enterprise business part of the known information, is represented by $\left\{z_{t}\right\}_{t=T+1}^{T+\Delta}$, at timet, $x_{t} \in R^{n}$, and $z_{t} \in R^{m}$, where $n$ and $m$ are characteristic dimensions. The output of business forecast of SMEs is $\Delta$ sales value after $T$. Therefore, the definition of business forecast of SMEs can be improved to the following formula:

$$
\left\{y_{t}\right\}_{t=T+1}^{T+\Delta}=F\left(\left\{x_{t}\right\}_{t=1}^{T},\left\{y_{t}\right\}_{t=1}^{T},\left\{z_{t}\right\}_{t=T+1}^{T+\Delta}\right)
$$




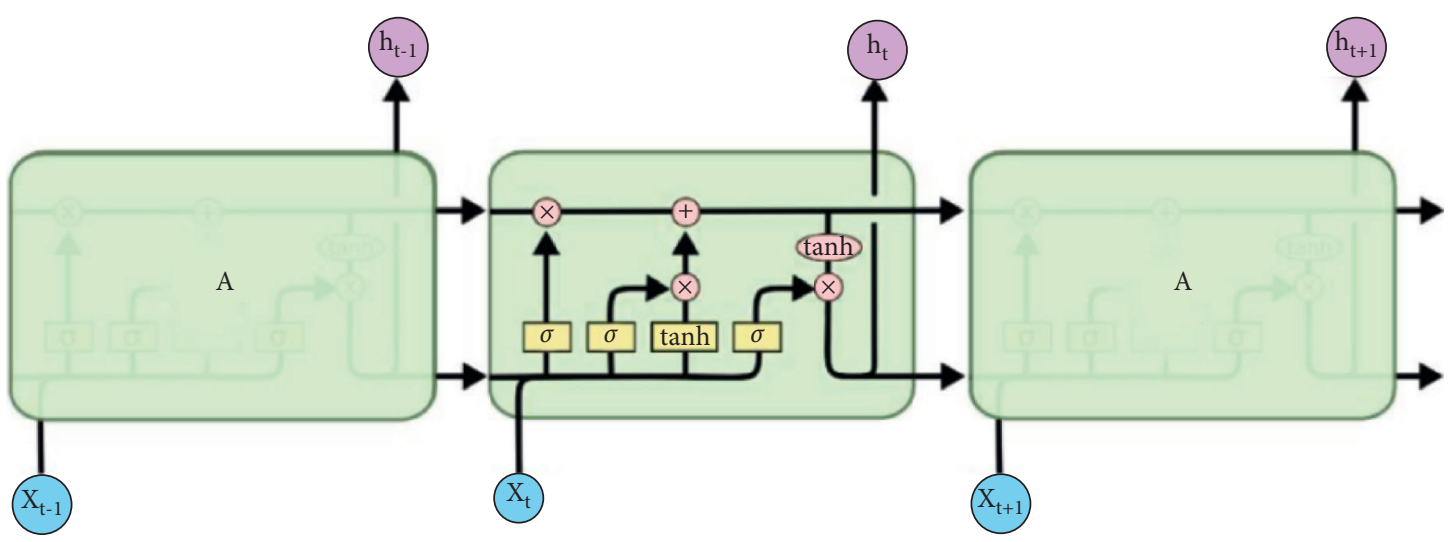

FIgURE 1: Internal structure of long- and short-term neural network [13].

where $\left\{x_{t}\right\}_{t=1}^{T}$ is the feature from 1 to $T$, and the feature dimension is $n$-dimension. $\left\{y_{t}\right\}_{t=1}^{T}$ indicates historical SME information. $\left\{z_{t}\right\}_{t=T+1}^{T+\Delta}$ is a partial known probability feature between $T+1$ and $T+\Delta$, and the feature dimension is $m$ dimension. $\left\{y_{t}\right\}_{t=T+1}^{T+\Delta}$ is the business forecast value of SMEs.

Due to the addition of some information, some changes will be made to the TADA model to adapt to the definition of the new SME business forecast model. In the previous model definition, the basic model is an encoder and decoder model, and some adjustments are made in the structure of the encoder and decoder. Firstly, the input features of the encoder stage are divided into internal feature input and external feature input, and the output of the hidden layer is obtained, respectively, after two LSTM algorithms. The output of the hidden layer is combined with the sales history of SMEs as the context vector to learn their joint representation, which constitutes the coding stage of the model. Because $\left\{z_{t}\right\}_{t=T+1}^{T+\Delta}$ cannot be used in the encoding phase of the model, no improvements are made to the encoder phase of the model. In the decoder stage, the original model uses the multiattention mechanism to obtain the input of the decoder stage, so the information of this part can be added in the decoding part for training. The formula for the decoder part is as follows:

$$
d_{t}^{\mathrm{con}}=\operatorname{LSTM}^{\mathrm{dec}}\left(x_{t}^{\mathrm{dec}}, d_{t-1}^{\mathrm{con}}\right),
$$

where $x_{t}^{\text {dec }}$ is the input of the LSTM model in the decoder stage. As part of the information is unknown and part of the information is known in the prediction period, a new method combining the characteristics of attention mechanism and some known features with probability is used. Among them, the characteristic output of attention mechanism is as follows:

$$
a_{t}^{\text {con }}=W_{\mathrm{dec}}\left[\sum_{t^{\prime}=1}^{T} a_{t t^{\prime}}^{\text {int }} h_{t^{\prime}}^{\text {int }} ; \sum_{t^{\prime}=1}^{T} a_{t t^{\prime}}^{\text {int }} h_{t^{\prime}}^{\text {ext }}\right]+b_{\mathrm{dec}}
$$

The specific explanation of the formula can be seen in the input of TADA model decoding stage above. $\left\{z_{t}\right\}_{t=T+1}^{T+\Delta}$ is added to the improved TADA model in the decoding stage, which is spliced with the results of the attention mechanism to obtain the input $x_{t}^{\text {dec }}$ in the decoder stage:

$$
x_{t}^{\mathrm{dec}}=\left[a_{t}^{\mathrm{dec}} ; z_{t}\right]
$$

In the decoder stage, the known features of current SME business are added, in which category features are embedded dimension reduction, and the weight vector of embedding dimension reduction is consistent with the weight of embedding dimension reduction input in the coding stage.

\section{Experiments}

To evaluate the effectiveness of the proposed system, we performed a number of experiments with different number of iterations. In this section, we will discuss the experimental setup and the results in detail.

\subsection{Experimental Parameter Design}

5.1.1. Data Set Partitioning. In order to compare the improved TADA algorithm with the TADA algorithm, the selection of data set, the division of training set and test set, and the evaluation indexes are as consistent as possible with the comparison method. In data set 1, 150 commodities were randomly selected from each enterprise's store for training, 20 commodities were used as the verification set, and 20 commodities were used as the test set. The data from August 16, 2016, to August 15, 2017, in the data set of each commodity were intercepted, and a total of 365 data pieces were trained and predicted. Data set 2 is the original data obtained. The training set, the verification set, and the test set use the full year 2020 data for each city in each province and are divided in an 8:1:1 ratio.

5.1.2. Evaluation Index. The evaluation index is measured by means of mean absolute error (MAE) and symmetric mean absolute percentage error (SMAPE). The formula of MAE is as follows:

$$
\text { MAE }=\frac{1}{N \times \Delta} \sum_{n=1}^{N} \sum_{t=T+1}^{T+\Delta}\left|y_{t}-y_{t}^{\prime}\right| .
$$

MAE has the advantage of being more intuitive, but it is more sensitive to outliers, which is the disadvantage of 
TABLE 1: Absolute errors of business value forecast by different methods.

\begin{tabular}{|c|c|c|c|}
\hline \multirow{2}{*}{ Number of iterations (times) } & \multicolumn{3}{|c|}{ The operating value of SMEs forecast absolute error (yuan) } \\
\hline & Method proposed by [7] & Method proposed by [8] & Our proposed method \\
\hline 10 & 3421.77 & 4367.63 & 122.32 \\
\hline 20 & 5473.22 & 3525.73 & 107.54 \\
\hline 30 & 5637.33 & 5435.53 & 89.54 \\
\hline 40 & 3452,87 & 3428.52 & 75.35 \\
\hline 50 & 2753.66 & 4356.71 & 84.75 \\
\hline 60 & 3655.32 & 3425.77 & 63.53 \\
\hline
\end{tabular}

TABLE 2: Absolute percentage error of business value forecast of SMSEs.

\begin{tabular}{|c|c|c|c|}
\hline \multirow{2}{*}{ Number of iterations (times) } & \multicolumn{3}{|c|}{ Absolute percentage error of SME business value forecast (\%) } \\
\hline & Method proposed by [7] & Method proposed by [8] & Our proposed method \\
\hline 10 & 56.87 & 54.76 & 2.86 \\
\hline 20 & 65.65 & 53.54 & 1.32 \\
\hline 30 & 65.87 & 48.65 & 3.87 \\
\hline 40 & 74.35 & 57.87 & 6.32 \\
\hline 50 & 71.06 & 60.54 & 5.64 \\
\hline 60 & 64.87 & 67.65 & 3.58 \\
\hline
\end{tabular}

mean absolute error. In order to solve the asymmetry of the mean absolute percentage error (MAPE), the symmetrical mean absolute percentage error (SMAPE) is formulated as follows:

$$
\mathrm{SMAPE}=\frac{100 \%}{N \times \mathrm{X}} \sum_{n=1}^{N} \sum_{t=T+1}^{T+\Delta} \frac{\left|y_{t}-y_{t}^{\prime}\right|}{\left|y_{t}\right|+\left|y_{t}^{\prime}\right|}
$$

If $y_{t}=y_{t}^{\prime}=0$, then SMAPE is formulated as follows:

$$
\text { SMAPE }=\frac{100 \%}{N \times \mathrm{X}} \sum_{n=1}^{N} \sum_{t=T+1}^{T+\Delta} 0,
$$

where $y_{t}$ and $y_{t}^{\prime}$ are the real and predicted values, respectively. The advantages of SMAPE are that it is always computable and formally symmetrical. The disadvantage is that the predicted value is too large in practice. However, comparisons can be made across data sets. It is also an advantage of SMAPE. Since the use of a single evaluation index has its specific shortcomings, this experiment will use MAE and SMAPE for evaluation.

\subsection{The Absolute Error of the Management Model Is MAE.} In order to verify the management effect of the model in this paper on SMEs, the method used in [7], the method in [8], and the method in this paper are used to predict the business value of SMEs with absolute error (MAE), and the experimental results are shown in Table 1. Meng et al. [7] focused on the problems of salary management and in SMEs such as unreasonable pay system. Dey et al. [8] focused on supply chain sustainability performance measurement and management for SMEs. Their framework determines the state of economic, social and environmental practices, and performance of the SMEs. Tables 1 and 2 present a detailed comparison of the results of the three systems.

By analyzing Table 1, we can see that the absolute errors of business value prediction of SMEs are different under different methods. When the number of iterations is 10 , the average absolute error of SME business value prediction of the method in literature [7] is 3421.77 yuan, the average absolute error of SME business value prediction of the method in literature [8] is 4367.63 yuan, and the average absolute error of SME business value prediction of this method is 122.32 yuan. When the number of iterations is 60 , the average absolute error of SME business value prediction of the method in literature [7] is 3655.32 yuan, the average absolute error of SME business value prediction of the method in literature [8] is 3425.77 yuan, and the average absolute error of SME business value prediction of this method is 63.53 yuan. The business value prediction error of SMEs in this method is far lower than that of other traditional methods, which shows that the business value prediction effect of SMEs in this method is better. The advantage of the proposed system over the other systems is that we have used state-of-the-art deep learning algorithms in our systems that are well known for enhancing the functionality and accuracy where used.

5.3. Average Absolute Percentage Error of Management Model. To verify the management effect of this model on SMEs, the methods of literature [7], literature [8], and the average absolute percentage error (SMAPE) of small- and mediumsized enterprise business value prediction of this method are used. The experimental results are shown in Table 2.

By analyzing Table 2, we can see that there are differences in the absolute percentage error of business value prediction of SMEs under different methods. When the number of iterations is 20 , the absolute percentage error of SME business value prediction of the method in literature [7] is $65.65 \%$, the absolute percentage error of SME business value prediction of the method in literature [8] is $53.54 \%$, and the absolute percentage error of SME business value prediction of this method is $1.32 \%$. When the number of iterations is 40 , 
the absolute percentage error of SME business value prediction of the method in literature [7] is $74.35 \%$, the absolute percentage error of SME business value prediction of the method in literature [8] is $57.87 \%$, and the absolute percentage error of SME business value prediction of this method is $6.32 \%$. When the number of iterations is 60 , the absolute percentage error of SME business value prediction of the method in literature [7] is $64.87 \%$, the absolute percentage error of SME business value prediction of the method in literature [8] is $67.65 \%$, and the absolute percentage error of SME business value prediction of this method is $3.58 \%$. This method always has a low absolute percentage error of business value prediction of SMEs, which shows that the management effect of this method is better.

\section{Conclusion}

This paper proposes a management model of SMEs based on the deep learning algorithm and analyzes the characteristics of SMEs. Based on the deep learning algorithm, the gating structure of small- and medium-sized enterprise management is designed, the internal structure diagram of long- and short-term neural network is given, the circular neural network model of small- and medium-sized enterprise management is constructed, the experimental indexes of the small- and medium-sized enterprise management model are designed, and the comparative experiment is carried out. The following conclusions are drawn through experiments:

(1) When the number of iterations is 60 , the average absolute error of the business value prediction of SMEs in this method is 63.53 yuan. The business value of SMEs predicted by this method has a good effect.

(2) When the number of iterations is 60 , the absolute percentage error of SME business value prediction is $3.58 \%$. The enterprise management model based on this method has a good management effect.

\section{Data Availability}

The data used to support the findings of this study are available from the corresponding author upon request.

\section{Conflicts of Interest}

The author declares that there are no conflicts of interest.
[3] X.-yong liu, "Study on the promotion of management accounting function of SMEs," Cooperative economy and science and technology, vol. 621, no. 22, pp. 156-157, 2019.

[4] Y.-tao Yang, "The application of internal control system in the management of SMEs," Executive, vol. 034, no. 002, pp. 62-63, 2020.

[5] R. Gruenbichler, J. Klucka, K. Haviernikova, and S. Strelcova, "Business performance management in small and mediumsized enterprises in the Slovak republic: an integrated threephase-framework for implementation," Journal of Competitiveness, vol. 13, no. 1, pp. 42-58, 2021.

[6] N. Niewhner, L. Asmar, F. Wortmann, D. Röltgena, A. Kühn, and R. Dumitrescu, "Design fields of agile innovation management in small and medium sized enterprises," Procedia CIRP, vol. 84, pp. 826-831, 2019.

[7] K. Meng, G. Lu, and M. Shen, "Research on the problems and countermeasures of salary management in SMEs," Human Resource Management - Select, vol. 1, no. 001, pp. 16-23, 2019.

[8] P. K. Dey, G. L. Yang, C. Malesios, D. De, and K. Evangelinos, "Performance management of supply chain sustainability in SMEs using a combined structural equation modelling and data envelopment analysis," Computational Economics, vol. 8, pp. 1-41, 2019.

[9] R. Cong, Research on Management Model and System Innovation of SMEs in China Based on Regional Economy, 2019.

[10] Y. Nie, Research on the Financial Services of Small and Medium-Sized Micro-enterprises from the Perspective of Big Data, 2019.

[11] Y. Chen, J. Xing, and X. Sun, "Problems and countermeasures of human resource management in small and medium sized enterprises," E3S Web of Conferences, vol. 235, no. 23, p. 01058, 2021.

[12] G. Produo, L. Parizotto, A. Tonso, and M. M. de Carvalho, "The challenges of project management in SMEs: a literature review based on bibliometric software and content analysis," Gestão \& Produção, vol. 27, no. 1, pp. 23-37, 2020.

[13] M. Yu, T. Quan, Q. Peng, X. Yu, and L. Liu, "A model-based collaborate filtering algorithm based on stacked AutoEncoder," Neural Computing \& Applications, vol. 6, 2021.

[14] R. Balcerzyk, "Knowledge management in SMEs," European Research Studies Journal, vol. 12, no. 03, pp. 529-544, 2020.

[15] B. Khadija and R. Mustapha, "Performance management of SMEs in Morocco," Business Management and Strategy, vol. 11, no. 3, pp. 66-78, 2020.

[16] A. Asad, M. F. Basheer, M. Irfan, and J. Jiang, "Open-Innovation and knowledge management in SMEs (SMEs): the role of external knowledge and internal innovation," Revista Argentina de Clinica Psicologica, vol. 12, no. 4, pp. 80-90, 2020.

[17] N. Jaimes and A. A. Marghoob, "Triage amalgamated dermoscopic algorithm," Journal of the American Academy of Dermatology, vol. 82, no. 6, pp. 1551-1552, 2020.

\section{References}

[1] C. Yu, "Research on process mechanism of enterprise management innovation based on technology acceptance Model," Scientific research management, vol. V40, no. 8, pp. 206-214, 2019.

[2] X. Yu, F. Jiang, J. Du, and D. Gong, "A cross-domain collaborative filtering algorithm with expanding user and item features via the latent factor space of auxiliary domains," Pattern Recognition, vol. 94, pp. 96-109, 2019. 